\title{
LOCALLY FINITE-DIMENSIONAL SETS OF OPERATORS
}

\author{
LEONYA LIVSHITS
}

(Communicated by Palle E. T. Jorgensen)

\begin{abstract}
For any pair of Banach spaces $V$ and $W$ a "global" description is given for the sets $S$ of operators in $B(V, W)$ satisfying the "local" condition that the linear span of the set $\{T(x) \mid T \in S\}$ is finite-dimensional for every $x$ in $V$.
\end{abstract}

\section{INTRODUCTION}

Definition 1. A collection $F$ of linear operators from a vector space $V$ to a vector space $W$ is said to be locally finite-dimensional whenever the linear span of the set $F_{x}=\{T(x) \mid T \in F\}$ is finite-dimensional for every $x$ in $V$.

$F$ is locally finite-dimensional exactly when $\operatorname{span}(F)$ is locally finite-dimensional because $\operatorname{span}\left(F_{x}\right)=(\operatorname{span}(F))_{x}$. (We write $\operatorname{span}(S)$ for the linear span of a set $S$.)

If $G \subset F$ and $F$ is locally finite-dimensional then $G$ is also locally finitedimensional.

If $V$ and $W$ are Banach spaces, we write $B(V, W)$ for the space of all linear operators from $V$ to $W$ that are bounded with respect to the usual norms on $V$ and $W$.

For each subset $\Omega$ of $B(V, W)$ we write $\Omega_{\mathrm{fin} . r a n k}$ for the subset of $\Omega$ consisting of those operators that are of finite rank.

Probably the most famous example of a "global" characterisation for sets of operators satisfying a given "local" property is that due to Kaplansky [1], which states that locally algebraic bounded operators on Banach spaces are algebraic. (Recently Larson [2] extended this result to the multivariate case.) A reductive algebra problem is possibly the best-known example of an open problem of the same kind.

In this note we present a "global" characterisation of locally finite-dimensional sets of bounded operators between two Banach spaces. This question arose naturally in the author's Ph.D. thesis [3] when continuity properties of Schur multiplication maps with respect to various topologies were considered.

\section{RESULTS}

Lemma 2. Let $F=\left\{R_{n} \mid n \in \mathbb{N}\right\}$ be a sequence of finite rank operators from a Banach space $V$ to a Banach space $W$. Then $F$ is locally finite-dimensional

Received by the editors January 21, 1992.

1991 Mathematics Subject Classification. Primary 47A05, 47A15.

(C) 1993 American Mathematical Society $0002-9939 / 93 \$ 1.00+\$ .25$ per page 
precisely when $\operatorname{span}\left(\bigcup_{n \in \mathbb{N}} \operatorname{Range}\left(R_{n}\right)\right)$ is finite-dimensional; that is, precisely when there exists a finite-dimensional subspace of $W$ containing all the ranges of operators in $F$.

Proof. The implication in one direction is obvious since $F_{x}$ is a subset of $\bigcup_{n \in \mathbb{N}} \operatorname{Range}\left(R_{n}\right)$. For the converse, suppose $F$ is locally finite-dimensional, but $\operatorname{span}\left(\bigcup_{n \in \mathbb{N}} \operatorname{Range}\left(R_{n}\right)\right)$ is infinite-dimensional. Then there is an infinite subsequence $G=\left\{G_{n} \mid n \in \mathbb{N}\right\}$ of $F$ such that $\operatorname{Range}\left(G_{1}\right) \subsetneq \operatorname{Range}\left(G_{1}\right)+$ Range $\left(G_{2}\right) \subsetneq \operatorname{Range}\left(G_{1}\right)+\operatorname{Range}\left(G_{2}\right)+\operatorname{Range}\left(G_{3}\right) \subsetneq \cdots$. (Sums here are vector space sums.) Denote $\operatorname{Range}\left(G_{1}\right)+\operatorname{Range}\left(G_{2}\right)+\cdots+\operatorname{Range}\left(G_{k}\right)$ by $M_{k}$. Suppose that there is an element $x$ of $V$ such that for every $k, G_{k+1}(x)$ is not in $M_{k}\left(G_{k+1}(x) \in \operatorname{Range}\left(G_{k+1}\right) \subset M_{k+1}\right)$. Then $\left\{G_{j}(x) \mid j \geq 2\right\}$ is a linearly independent set because $\operatorname{span}\left(\left\{G_{j}(x) \mid 2 \leq j \leq k\right\}\right) \subset M_{k}$ for every $k$, while $G_{k+1}(x)$ is not in $M_{k}$. Thus $\operatorname{span}\left(\left\{G_{j}(x) \mid 2 \leq j \leq k\right\}\right)$ is infinite-dimensional, which is in contradiction with the assumptions. Therefore no such $x$ exists. So the intersection of the sets $G_{k+1}^{-1}\left(M_{k}^{\mathrm{c}}\right)$ is empty (here the superscript c stands for the set theoretic complement and the superscript -1 stands for the preimage under the given map). Now $\varnothing_{V}=\bigcap_{k \in \mathbb{N}} G_{k+1}^{-1}\left(M_{k}^{c}\right)=\bigcap_{k \in \mathbb{N}}\left(G_{k+1}^{-1}\left(M_{k}\right)\right)^{\mathbf{c}}=$ $\left(\bigcup_{k \in \mathbb{N}} G_{k+1}^{-1}\left(M_{k}\right)\right)^{\mathbf{c}}$, and therefore we can conclude that $\bigcup_{k \in \mathbb{N}} G_{k+1}^{-1}\left(M_{k}\right)=V$. Yet each $G_{k+1}^{-1}\left(M_{k}\right)$ is a preimage of a finite-dimensional subspace of $W$ under a continuous map and, consequently, must be a closed subspace of $V$. By Baire Category Theorem there exists an integer $k_{0}$ such that $G_{k_{0}+1}^{-1}\left(M_{k_{0}}\right)=V$; that is, there exists an integer $k_{0}$ such that $\operatorname{Range}\left(G_{k_{0}+1}\right) \subset M_{k_{0}}$. This is clearly in contradiction with the choice of the $G_{k}$ 's and the construction of the $M_{k}$ 's and so the proof is complete.

The following theorem clearly follows.

Theorem 3. A set $F$ of finite rank operators from a Banach space $V$ to a Banach space $W$ is locally finite-dimensional if and only if $\operatorname{span}\left(\bigcup_{R \in F} \operatorname{Range}(R)\right)$ is finite dimensional.

Definition 4. We say that a subalgebra $A$ of $B(V, V)$ is strongly generated by operators of finite rank if the strong closure of $A_{\text {fin.rank }}$ is equal to $A$. If $V$ is a Hilbert space then for any algebra $A$ strongly generated by operators of finite rank, the weak closure of $A_{\text {fin.rank }}$ is also equal to $A$.

Corollary 5. Let $A$ be a subalgebra of $B(V, V)$ strongly generated by operators of finite rank. Then $A$ has an infinite-dimensional cyclic invariant linear manifold (subspace) if and only if for each finite-dimensional subspace $N$ of $W$ there exists $x$ in $V$ and an operator $T$ in $A$ such that $T(x)$ is not in $N$; that is, if and only if for every finite-dimensional subspace $N$ of $W, A$ is not a subalgebra of $\{S \in B(V, V) \mid \operatorname{Range}(S) \subset N\}$.

Proof. A subalgebra of $B(V, W)$ has no infinite-dimensional cyclic invariant linear manifolds exactly when it is locally finite-dimensional. If $A$ has this property then so does the algebra $A_{\text {fin.rank }}$. Consequently, by Theorem 3 there exists a finite-dimensional subspace $N$ of $W$ such that $N$ contains the ranges of all operators in $A_{\text {fin.rank }}$. Then $N$ must contain the ranges of all operators in $A$ since $A_{\text {fin.rank }}$ is strongly dense in $A$ and $N$ is closed. This completes the 
proof of the implication in one direction. The implication in the other direction is clear.

Theorem 6 (Larson [2]). Let $V$ and $W$ be Banach spaces, and let $G$ be a linear submanifold of $B(V, W)$. If the dimension of $G$ is at most countably infinite and if $G_{\mathrm{fin} . \mathrm{rank}}=\{0\}$, then there exists a separating vector for $G$ in $V$.

Theorem 7. A subset $S$ of $B(V, W)$ is locally finite-dimensional if and only if there exist a finite-dimensional subspace $L$ of $B(V, W)$ (containing no operators of finite rank) and a finite-dimensional subspace $N$ of $W$ such that $S$ is contained in the vector space sum $L+\{T \in B(V, W) \mid \operatorname{Range}(T) \subset N\}$. Moreover, $N$ can always be taken equal to $\operatorname{span}\left(\bigcup_{T \in S_{\text {fin.rank }}} \operatorname{Range}(T)\right)$. If $V$ and $W$ are Hilbert spaces, then $L$ can always be taken to be a subspace of $\left\{T \in B(V, W) \mid \operatorname{Range}(T) \subset N^{\perp}\right\}$.

Proof. Without loss of generality we may assume that $S$ is a linear manifold.

We assume the linear dimension of $S$ to be infinite because if $S$ is finitedimensional the result follows trivially.

Use $\operatorname{span}\left(\bigcup_{T \in S_{\text {fin.rank }}} \operatorname{Range}(T)\right)$ as $N$. Note that $N$ is then finite-dimensional by Theorem 3 since $S_{\text {fin.rank }}$ is locally finite-dimensional (being a subset of $S$ ). Let $L$ be any vector space complement of $S_{\text {fin.rank }}$ in $S$; that is, $L(+) S_{\text {fin.rank }}=$ $S$, where $(+)$ indicates the disjoint vector space sum. (Note that such $L$ can always be constructed via an extension of a basis of $S_{\text {fin.rank }}$ to a basis of $S$, since there are no imposed topological requirements.) $L$ contains no operators of finite rank. Suppose $L$ is infinite-dimensional. Pick any countably infinitedimensional linear submanifold $D$ of $L$, and observe that $D$ does not have a separating vector in $V$ since $D$ is locally finite-dimensional $\left(D_{x}\right.$ is finitedimensional for every $x$ in $V$; yet if $x_{0}$ is a separating vector for $D$ then the dimensions of $D$ and $D_{x_{0}}$ must be the same). Therefore by Theorem $6, D$ (and thus $L$ ) must contain a nonzero operator of finite rank. This is a contradiction to the construction of $L$. Thus $L$ is finite-dimensional. The proof of this part of the theorem is completed by observing that from the definition of $N$, $S_{\text {fin.rank }}$ is contained in $\{T \in B(V, W) \mid \operatorname{Range}(T) \subset N\}$.

In the case where $V$ and $W$ are both Hilbert spaces, pick any basis $\left\{E_{1}, E_{2}\right.$, $\left.E_{3}, \ldots, E_{t}\right\}$ of $L$, and observe that there are operators $Q_{1}, Q_{2}, Q_{3}, \ldots, Q_{t}$ in $B(V, W)$ with ranges lying in $N$, such that the ranges of $E_{1}+Q_{1}, E_{2}+Q_{2}$, and $E_{3}+Q_{3}, \ldots, E_{t}+Q_{t}$ are all in $N^{\perp}$. Let $\widetilde{L}$ be the linear span of $\left\{E_{1}+Q_{1}, E_{2}+Q_{2}, E_{3}+Q_{3}, \ldots, E_{t}+Q_{t}\right\}$ within $B(V, W)$. Note that $\widetilde{L}$ is a finite-dimensional subspace of $B(V, W)$ containing only operators whose ranges lie in $N^{\perp}$. Also $\widetilde{L}(+)\{T \in B(V, W) \mid \operatorname{Range}(T) \subset N\}=L(+)\{T \in$ $B(V, W) \mid \operatorname{Range}(T) \subset N\}$. Taking $\widetilde{L}$ instead of $L$ provides us with the required result.

Corollary 8. If a linear submanifold $S$ of $B(V, W)$ is locally finite-dimensional, then $S_{\text {fin.rank }}$ has finite codimension in $S$.

Corollary 9. Let $A$ be a norm closed subalgebra of $B(V, V)$. If $A$ does not have any infinite-dimensional cyclic invariant subspaces, then the linear codimension of $A_{\text {fin.rank }}$ in $A$ is finite.

The property of being locally finite-dimensional is defined in purely algebraic terms, yet the theorems above contain assumptions about the topology of the 
underlying spaces. It is natural to ask whether the same results hold with no topological assumptions (and therefore with different proofs). The answer to this question is (surprisingly enough) negative as the following example, proposed by Hadwin, demonstrates.

Example 10. Let $V$ be any infinite-dimensional complex vector space, and let $E$ be a basis of $V$. Let $S$ be the manifold of all linear transformations on $V$ having the elements of $E$ as eigenvectors. Then $S$ is locally finite dimensional, but there does not exist a finite-dimensional subspace $M$ of the space $L(V)$ of all linear transformations on $V$ and a finite-dimensional subspace $N$ of $V$, such that $S \subset M+L(V, N)$ (here we write $L(V, N)$ for $\{T \in L(V) \mid \operatorname{Range}(T) \subset N\})$.

Proof. $S$ is locally finite-dimensional because every vector in $V$ is a linear combination of finitely many basis vectors in $E$, and consequently the span of these same basis elements contains all the images of the given vector under the linear transformations in $S$.

To complete the proof one can use Corollary 8 and demonstrate that the space of all finitely nonzero sequences of complex numbers has infinite codimension in the space of all infinite sequences of complex numbers. Alternatively, let $N$ be any finite-dimensional subspace of $V$. Form quotient vector space $V / N$, and note that $E+N$ is a spanning set for $V / N$. Thus there exists a subset $F$ of $E$ such that $F+N$ is the basis of $V / N$. Since $N$ has infinite codimension in $V$, we conclude that $F$ is infinite. Therefore the space of operators in $S$ that vanish on the complement of $F$ in $E$ is infinite-dimensional. It follows that the dimension of the quotient space $S /(S \cap L(V, N))$ is infinite, and consequently there does not exist a finite-dimensional subspace $M$ such that $S$ is contained in the vector space sum $M+L(V, N)$. Since $N$ was chosen arbitrarily, the proof is complete.

In conclusion we observe that even though the property of being locally finitedimensional is preserved under taking spacially induced Banach space isomorphisms, it is not preserved in general under taking (even isometric) Banach space isomorphisms. Indeed, let $H$ be a separable Hilbert space with a fixed basis $\left\{e_{i} \mid i \in \mathbb{N}\right\}$, and let $S_{1}$ stand for the algebra $\left\{T \in B(H) \mid e_{1}\right.$ spans $\left.\operatorname{Range}(T)\right\}$ while $S_{2}$ stands for $\left\{T \in B(H) \mid e_{1}\right.$ spans $\left.\operatorname{Range}\left(T^{t}\right)\right\}$, where $T^{t}$ is the operator in $B(H)$ defined by $\left\langle T^{t}\left(e_{i}\right), e_{j}\right\rangle=\left\langle T\left(e_{j}\right), e_{i}\right\rangle$, for all $i$ and $j$. The map $\Psi$ on $B(H)$, defined by $\Psi(T)=T^{t}$, is an isometric Banach space isomorphism which maps $S_{1}$ to $S_{2} . S_{1}$ is clearly locally finite-dimensional, but $S_{2}$ is not, since for every vector $x$ in $H$ there is an element $T$ of $S_{2}$ such that $T\left(e_{1}\right)=x$.

\section{ADDED IN PROOF}

The author would like to note that if $V$ and $W$ are any Banach spaces then $L$ in Theorem 7 can always be taken to be a subspace of the space $\{T \in$ $B(V, W) \mid \operatorname{Range}(T)$ is a subspace of a closed complement of $N$ in $W\}$.

\section{ACKNOWLEDGMENTS}

The author thanks A. Markus, S. Rosenoer, and M. Lacruz for the stimulating discussions we had on this subject. 


\section{REFERENCES}

1. I. Kaplansky, Infinite abelian groups, Univ. of Michigan Press, Ann Arbor, MI, 1954.

2. D. R. Larson, Reflexivity, algebraic reflexivity and linear interpolation, Amer. J. Math. 110 (1988), 283-299.

3. L. Livshits, Generalised Schur products for matricies with operator entries, Ph.D. Thesis, Univ. of Toronto, 1991.

Department of Mathematics, Central Michigan University, Mount Pleasant, MichiGAN 48859

Current address: Department of Mathematics, Bishop's University, Lennoxville, Quebec, Canada J1M $1 \mathrm{Z7}$ 